\title{
Thermal Analysis and Simulation of the Superconducting Magnet in the SpinQuest Experiment at Fermilab
}

\author{
Z. Akbar* \\ University of Virginia \\ E-mail: za2hdevirginia.edu \\ D. Keller \\ University of Virginia \\ E-mail: dustin@jlab.org
}

\begin{abstract}
The SpinQuest experiment at Fermilab aims to measure the Sivers asymmetry for the $\bar{u}$ and $\bar{d}$ sea quarks in the range of $0.1<x_{B}<0.5$ using the Drell-Yan production of dimuon pairs. A nonzero Sivers asymmetry would provide an evidence for a nonzero orbital angular momentum of sea quarks. The proposed beam intensity is $1.5 \times 10^{12}$ of $120 \mathrm{GeV}$ unpolarized proton/sec. The experiment will utilizes a target system consisting of a 5T superconducting magnet, transversely polarized $\mathrm{NH}_{3}$ and $\mathrm{ND}_{3}$ targets, a ${ }^{4} \mathrm{He}$ evaporation refrigerator, a $140 \mathrm{GHz}$ microwave source and a large pumping system. The expected average of the target polarization is $80 \%$ for the proton and $32 \%$ for the deuteron. The polarization will be measured with three NMR coils per target cell. A quench analysis and simulation in the superconducting magnet are performed to determine the maximum intensity of the proton beam before the magnet become resistive. The simulation of quenches in the superconducting magnets is a multiphysics problem of highest complexity. The heat transfer from metal to helium goes through different transfer and boiling regimes as a function of temperature, heat flux, and transferred energy. All material properties are temperature dependent. A GEANT based simulation is used to calculate the heat deposited in the magnet and the subsequent cooling processes are modeled using the COMSOL Multiphysics.
\end{abstract}

The 18th International Workshop on Polarized Sources, Targets, and Polarimetry, PSTP2019

23-27 September, 2019

Knoxville, Tennessee

${ }^{*}$ Speaker. 


\section{Introduction}

One of the central question in nuclear physics is how the nucleon's spin is built up from its quark and gluon constituents. Recent theoretical developments have provided the decomposition of the nucleon spin into its quark and gluon contributions and further separation into their spin and orbital components. According to proton angular momentum sum rule [1]

$$
\frac{1}{2}=\frac{1}{2} \Delta \Sigma+\Delta G+L_{q}+L_{q}
$$

where $\Delta \Sigma$ denotes the combined quark and antiquark spin contribution, $L_{q, g}$ are the quark and gluon orbital angular momentum contributions and $\Delta G$ can be interpreted as gluon spin contribution. Experiments on polarized Deep Inelastic Scattering (DIS) showed that the quark and antiquark spins $(\Delta \Sigma)$ only contributes $\sim 0.25$ of the proton spin [2]. The recent experimental data from RHIC also showed that the sum of both quark and gluon spin contributions still cannot account for the rest of the proton spin [3]. The large missing fraction of the spin is likely to be carried by the orbital angular momentum (OAM) of the quarks and gluons [4].

One way to extract the quark OAM $L_{q}$ is through Transverse Momentum Distribution (TMD). One Among the eight TMDs, the sivers function, represents the correlation of the transverse momentum of an unpolarized parton with the spin of a transversely polarized nucleon [5]. A non-zero Sivers asymmetry requires the existence of the parton OAM. The experimental data from the SemiInclusive Deep Inelastic Scattering (SIDIS) experiments indicate that the OAM of the $\mathrm{d}$ and $\mathrm{u}$ quarks are approximately equal and opposite in sign [6] [7] [8]. Thus, the valence quarks may carry little net of OAM. The Spinquest experiment at Fermilab aims to perform a measurement of the Sivers asymmetry for sea quarks, which the data are notably lacking using Drell-Yan production from an unpolarized $120 \mathrm{GeV}$ proton beam interacting with transversely polarized $\mathrm{NH}_{3}$ and $\mathrm{ND}_{3}$ targets [9].

The polarized-target system consist of a $5 \mathrm{~T}$ superconducting split coil magnet, a ${ }^{4} \mathrm{He}$ evaporation refrigerator, a $140 \mathrm{GHz}$ microwave source and a large $15000 \mathrm{~m}^{3} / \mathrm{hr}$ pumping system. Loss of superconducting state, namely quench, is an undesirable phenomenon that occurs when parts of the superconducting magnet become resistive. It is a situation that we want to prevent from happening due to lengthy recovery and liquid helium loss. The quench event has to be understood and avoided. For this, a proper understanding of how the magnet temperature increase due to the external heat source is essential. A high-intensity of $120 \mathrm{GeV}$ proton beam (up to $1.5 \times 10^{12}$ proton/sec) will be delivered to the polarized target. The heat generated in the superconducting magnet from the beam-target interactions is taken away by the liquid helium. However if the rate of heat generation in the superconducting magnet persists to be greater than the rate of heat transfer to the liquid helium the temperature of the magnet increases and might exceed the critical surface. Therefore a proper thermal analysis of the heat-load in the superconducting magnet coils is required to study what maximum proton beam intensity the experiment could achieve without quenching. 


\section{Thermal Analysis of the Superconducting Magnet}

The basic element of the coils is the superconducting wire or strand where tiny ( $\sim \mu \mathrm{m})$ NbTi filaments are bundled together in several hexagons and embedded in a copper matrix. The superconducting coils are then surrounded by 316LN stainless steel and impregnated with epoxy to prevent them from moving due to the enormous amount of Lorentz force when the magnet is energized. Figure 1 shows a type of split-pair magnet used in JLAB [10].

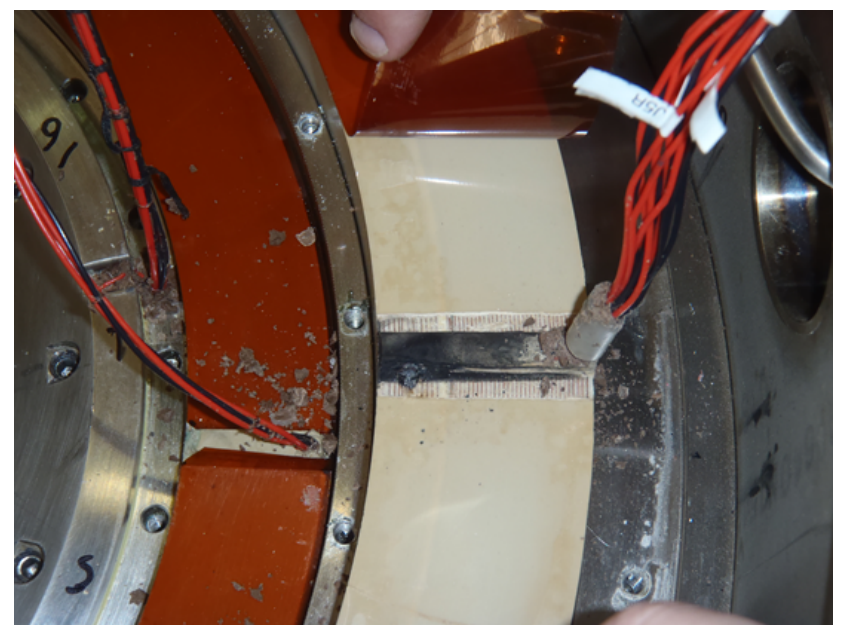

Figure 1: The superconducting wires impregnated in epoxy and further protected by $316 \mathrm{LN}$ stainless steel. Image source [10]

The thermal processes within the superconducting magnet are governed by a general heat transfer equation

$$
c(T) \frac{\partial T}{\partial t}=\nabla(\kappa(T) \nabla T)+P_{e x t}+P_{H e},
$$

where $c(T)$ is the volumetric heat capacity and $\kappa(T)$ is the thermal conductivity. $P_{\text {ext }}$ is the heat flux from external heat sources per unit volume and $P_{H e}$ is the heat flow to the liquid Helium.

The external heat sources in the superconducting magnet mainly come from the beam-target interaction. The collimator focus the $120 \mathrm{GeV}$ proton beam with the distribution profile shown in figure 2 so there is no direct beam-magnet interaction, but the secondary particle produced from the beam-collimator as well as beam-target interactions (figure 3) during the $4.4 \mathrm{~s}$ of the beam spill deposited a non-negligible heat that could possibly quench the magnet.

The amount of the heat deposited to the superconducting magnet from the beam-target and beam-collimator interactions were obtained form the Geant simulation. Figure 4 shows the average heat load for each slices of the superconducting magnet.

The magnet coils are immersed in the liquid helium reservoir which has a temperature of 4.2 Kelvin at 1 Atm. A KNF-N0150 pump with ultimate vacuum pressure of $100 \mathrm{mbar}$ will be used to pump the helium vapor out of the reservoir. According to helium-phase diagram, shown in figure 9, a pressure of $100 \mathrm{mbar}$ correspond to the liquid-helium temperature of $2.5 \mathrm{~K}$. The heat transferred into the liquid helium goes through several different regimes, depend on the phase of the liquid. At temperature of $2.5 \mathrm{~K}$ and pressure of $100 \mathrm{mbar}$ the liquid helium exist as normal fluid (He-I). 


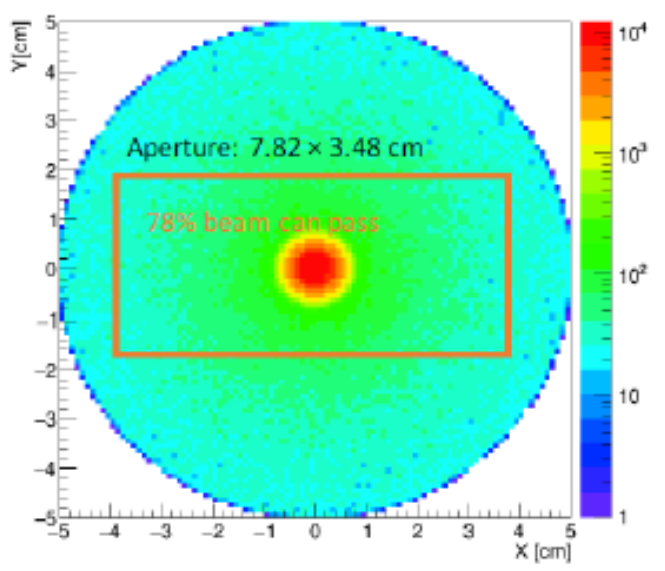

Figure 2: The Gaussian plus tail distribution of the proton-beam profile. The collimator allows $78 \%$ of the beam passed to the target.

The processes which are relevant in the normal-fluid phase (He-I) are natural convection, nucleate boiling, and film boiling regimes.

Depending on the heating power, the heat transfer will start in the natural convection regime. It is followed by the nucleate boiling regime and finally it enters the film boiling regime. Below a few $\mathrm{W} / \mathrm{m}^{2}$ of heat flux to the liquid helium there is no phase change and cooling is assumed to be natural convection,

$$
h\left(T_{c}, T_{H e}\right)=a_{N C}\left(T_{c}-T_{H e}\right),
$$

where $T_{c}$ is the temperature of the conductor surface, $T_{H e}$ is the temperature of the Helium, and the typical value of $a_{N C}$ is $500 \mathrm{~W} /\left(\mathrm{m}^{2} \mathrm{~K}\right)$. As the heat flux crosses the limit of natural convection (10 $\mathrm{W} / \mathrm{m}^{2}$ ), helium vapor is formed on the surface of the conductor. Then bubbles are formed which the rate of growth increase as the heat flux increase. As the bubbles detach from the surface, the cold liquid rush down to cool the surface. The amount of the heat flow for this nucleate boiling process is

$$
h\left(T_{c}, T_{H e}\right)=a_{N B}\left(T_{c}-T_{H e}\right)^{2.5},
$$

where the typical value of $a_{N B}$ is $50000 \mathrm{~W} /\left(\mathrm{m}^{2} \mathrm{~K}^{-2.5}\right)$. At a higher rate of the heat flux, the bubbles become unstable and they form a layer of helium vapor, preventing the liquid helium from being a

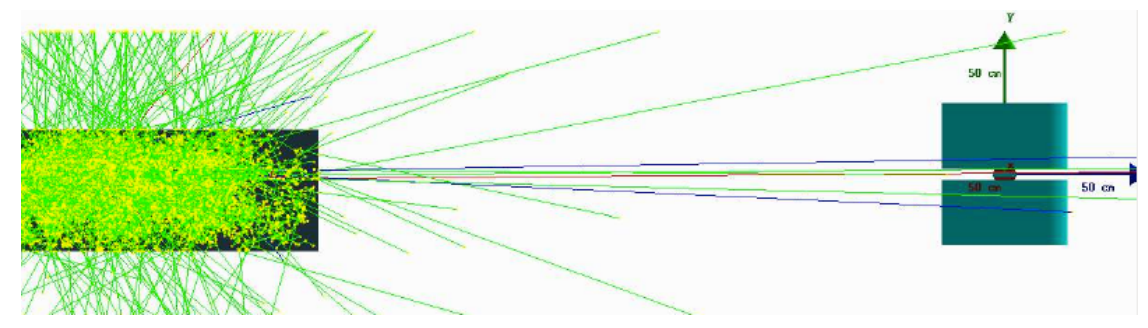

Figure 3: The illustration of the beam-target and the beam-collimator interactions. 
direct contact with the conductor. The heat flow for this film-boiling process is given by

$$
h\left(T_{c}, T_{H e}\right)=a_{F B}\left(T_{c}-T_{H e}\right),
$$

where the typical value of $a_{F B}$ is $250 \mathrm{~W} /\left(\mathrm{m}^{2} \mathrm{~K}\right)$. The steady state of film-boiling regime is reached after approximately 0.1 second. Since The proton beam for the SpinQuest experiment run continuously for 4.4 second per spill, the film-boiling process (equation 2.4) is a applied to simplify the analysis.

In our model the superconducting wire is considered as a uniformly mixture of the NbTi filaments and copper matrix with effective thermal properties. We use the Rayleigh model to determine the thermal conductivity of the superconducting-wire composite [15]. This model based on a continuous matrix reinforced with parallel cylindrical fillers arranged in uniaxial simple cubic array.

Thermal conductivity of the composite is directional dependent. If $z$ is the axis of the filler (NbTi), the effective thermal conductivity in the longitudinal direction is

$$
\kappa_{e f f}, z=\kappa_{C u}+\left(\kappa_{N b T i}-\kappa_{C u}\right) \phi,
$$

and the effective thermal conductivity in the transverse direction is

$$
\kappa_{e f f}, T=\kappa_{C u}+\frac{2 \kappa_{C u} \phi}{C_{1}-\phi+C_{2}\left(0.30584 \phi^{4}+0.013363 \phi^{8}+\ldots\right)},
$$

where $\phi$ is the volume fraction of the $\mathrm{Nb}-\mathrm{Ti}$ (we use $\phi=0.4$ ), and

$$
C_{1}=\frac{\kappa_{N b T i}+\kappa_{C u}}{\kappa_{N b T i}-\kappa_{C u}}
$$
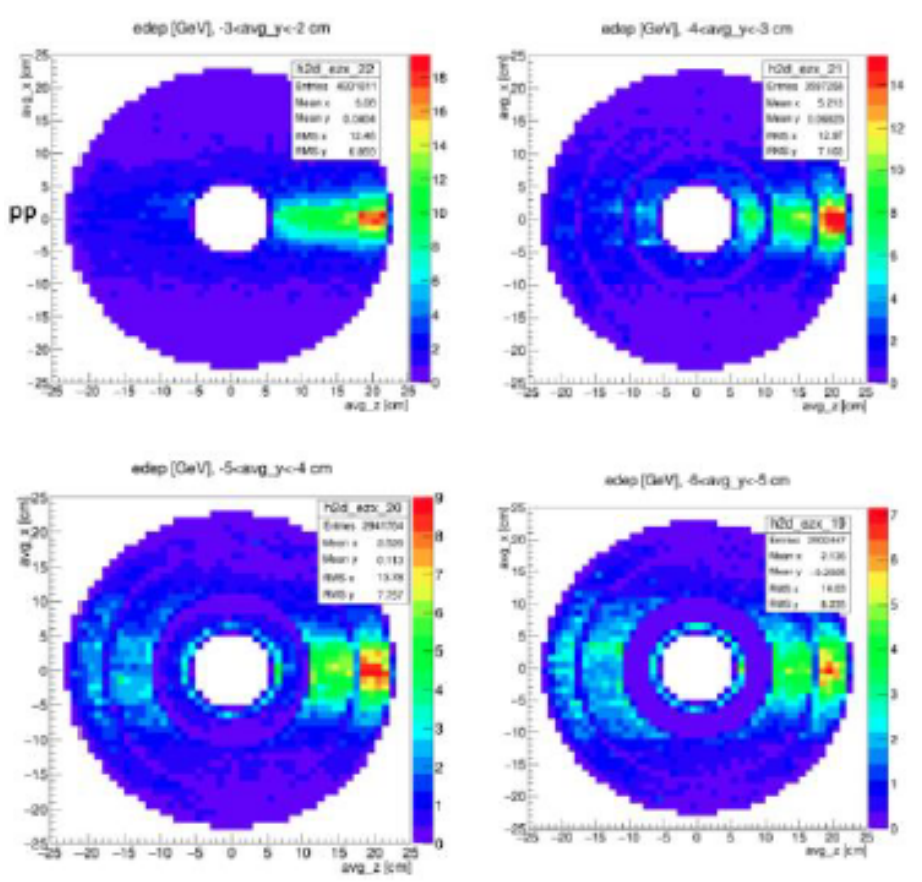

Figure 4: The heat deposited inside the magnet coils obtained from the Geant simulations. 


$$
C_{2}=\frac{\kappa_{N b T i}-\kappa_{C u}}{\kappa_{N b T i}+\kappa_{C u}} .
$$

The specific heat of the superconducting wire is also obtained from the specific heat of $\mathrm{NbTi}$ $\left(C_{N b T i}\right)$ and copper $\left(C_{C u}\right)$,

$$
C_{s c}=\phi C_{N b T i}+(1-\phi) C_{C u} .
$$

The superconducting wires are impregnated in epoxy and then surrounded by $316 \mathrm{LN}$-stainless steel as shown in figure 1. The top surface of the magnet and the stainless steel are in a direct contact with the liquid helium. Therefore, the film-boiling processes are only happen at the top surface of the magnet and the $316 \mathrm{LN}$-stainless steel.

\section{Finite Element Method of Simulation using COMSOL Multiphysics}

The objective of this study is to calculate the maximum intensity of the proton beam before the magnet quench. The equations governing the heat transfer are solved using Finite Element Method (FEM), implemented in COMSOL Multiphysics software. The FEM subdivides a large system into smaller, simpler parts called finite elements by the construction of a mesh of the object. The equations that model these finite elements are then assembled into a larger system of equations that models the entire problem.

The critical temperature of the superconducting wire as a function of magnetic field $T_{c}(B)$ is

$$
T_{c}(B)=T_{c 0}\left(1-\frac{B}{B_{c 20}}\right)^{0.59}
$$

where $T_{c 0}$ and $B_{c 20}$ for the NbTi superconducting wire is $T_{c 0}=9.2 \mathrm{~K}$ and $B_{c 20}=14.5 \mathrm{~T}$ [13]. Since the critical temperature depends on the magnetic field, the magnetic field inside the superconducting magnet need to be determined precisely. The Magnetic field in the target area between the coils, measured using Nuclear Magnetic Resonances is $5 \mathrm{~T}$ with the homogeneity level in the order of $10^{-4}$. The magnetic field outside the magnet reservoir is also measured using a handheld-Lakeshore Gauss meter. However, there is no information about the magnetic field inside the magnet itself.

These measurements could be extrapolated to build a three-dimensional map of the magnetic field inside and around the superconducting magnet. Assuming static current and field, the magnetic vector potential A must satisfy the following equation:

$$
\nabla \times\left(\mu^{-1} \nabla \times \mathbf{A}\right)=\mathbf{J}
$$

where $\mu$ is the permeability, and $\mathbf{J}$ denotes the applied current density. The relations between the magnetic field $\mathbf{H}$, the magnetic flux density $\mathbf{B}$, and the potential are given by

$$
\mathbf{B}=\nabla \times \mathbf{A}
$$

and

$$
\mathbf{H}=\mu^{-1} \mathbf{B} .
$$

The geometry of the superconducting magnet consist of three pairs of coils which form a modified Maxwell coil. A Maxwell coil is a device for producing a large region of nearly uniform magnetic field. Maxwell coil is an improvement of Helmholtz coil, with a better field uniformity but 
at the expense of more material and complexity. The set of partial differential equations are solved using COMSOL Multiphsics inside and outside the coils. Figure 5 shows the mesh construction of the model where tetahedral is used as the basic shape of the finite elements.

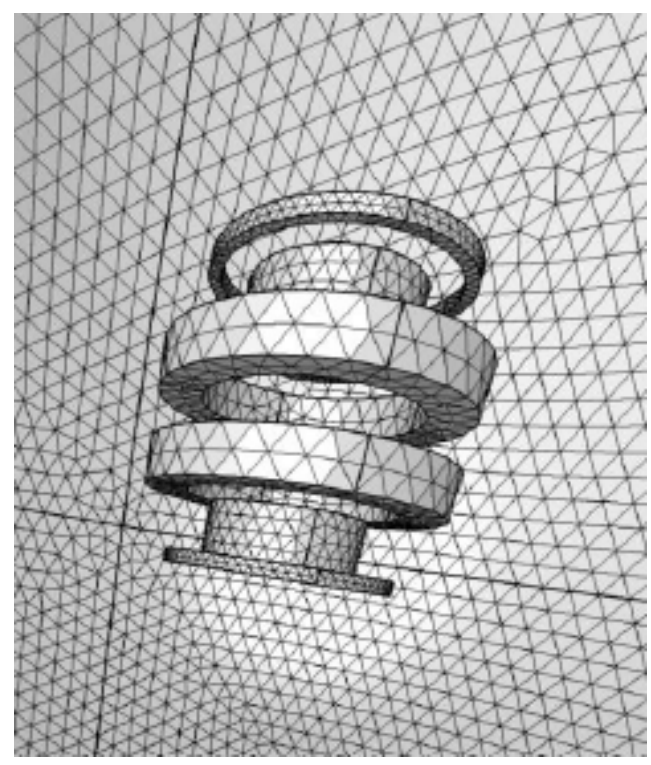

Figure 5: The construction of the tetahedral mesh for the magnet model.

The next step of the simulation is thermal simulation which aims to build a temperature profile inside the magnet as a function of time for a particular beam intensity. The temporal profile of the beam is one of the important input for this simulation along with the thermal properties of the materials and the heat transfer processes. The proton beam will be delivered in a spill which is 4.4 second long every minute. The time structure of the beam is illustrated in figure 6 below.

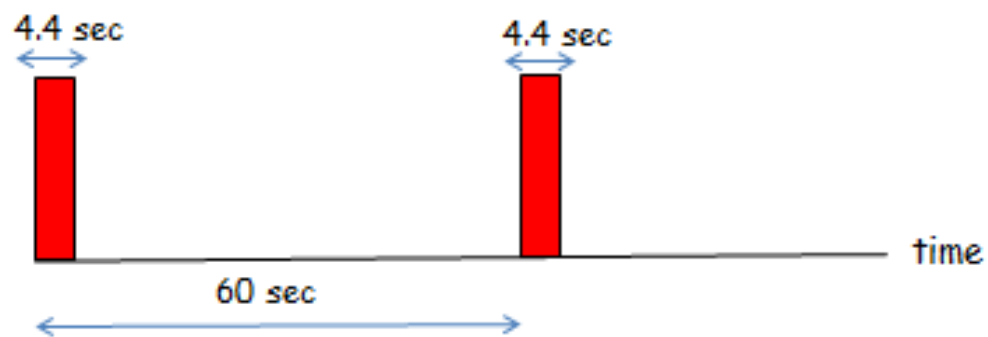

Figure 6: The time structure of the proton beam showing 4.4 second long per spill.

\section{Results and Discussion}

Figure 7 shows the magnetic field in the magnet obtained from the simulation. The maximum field is $6.85 \mathrm{~T}$ and according to the equation 3.1 the critical temperature of the magnet is $6.3 \mathrm{~K}$. To verify the validity of this results, the simulation and measurement results were compared. Table 1 shows the agreement of the magnetic-field strength at target area obtained from the measurements and the simulation. 


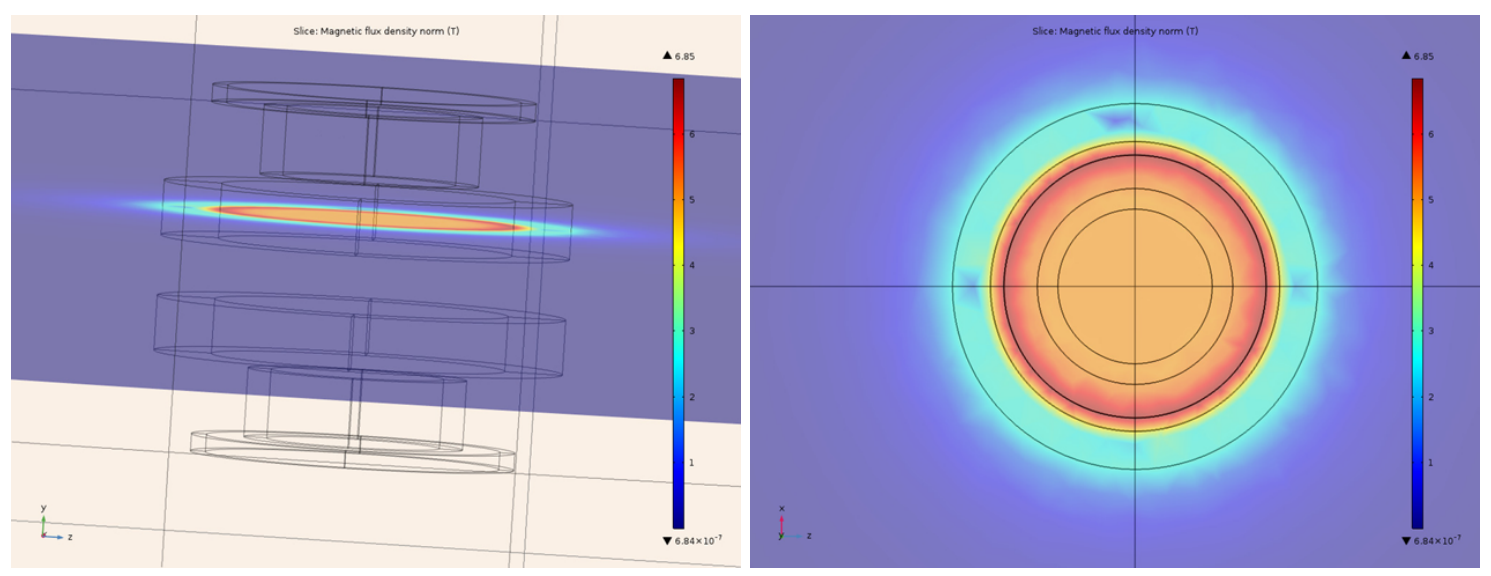

Figure 7: The magnetic field distribution inside the superconducting magnet. The maximum field is $6.85 \mathrm{~T}$.

Table 1: The value of the magnetic field in the target area obtained from the measurements and simulation

\begin{tabular}{cccc}
\hline$z(\mathrm{~cm})$ & $y(\mathrm{~cm})$ & Measurement $(\mathrm{T})$ & Simulation $(\mathrm{T})$ \\
\hline$-3.75-3.75$ & 0.0 & $5.0195-5.0199$ & $5.0192-5.0197$ \\
\hline 0.0 & $-2.0-2.0$ & $5.0196-5.0197$ & $5.0195-5.0197$ \\
\hline 3.75 & $-2.0-2.0$ & $5.0190-5.0197$ & $5.0191-5.0204$ \\
\hline
\end{tabular}

Before we turn on the KNF-N0150 pump the liquid helium temperature is $4.2 \mathrm{~K}$. Figure 8 shows the temperature distribution at the end of the first beam spill, $t=4.4 \mathrm{~s}$ in the magnet and the former with the beam intensity of $1 \times 10^{12}$ proton/sec and no pumping on the magnet reservoir. The hot spot in the magnet are spread uniformly due to the high thermal conductivity of the chopper matrix. The maximum temperature in the magnet is $6.1 \mathrm{~K}$. The hot spots are still exist in the stainless steel due to its lower thermal conductivity.

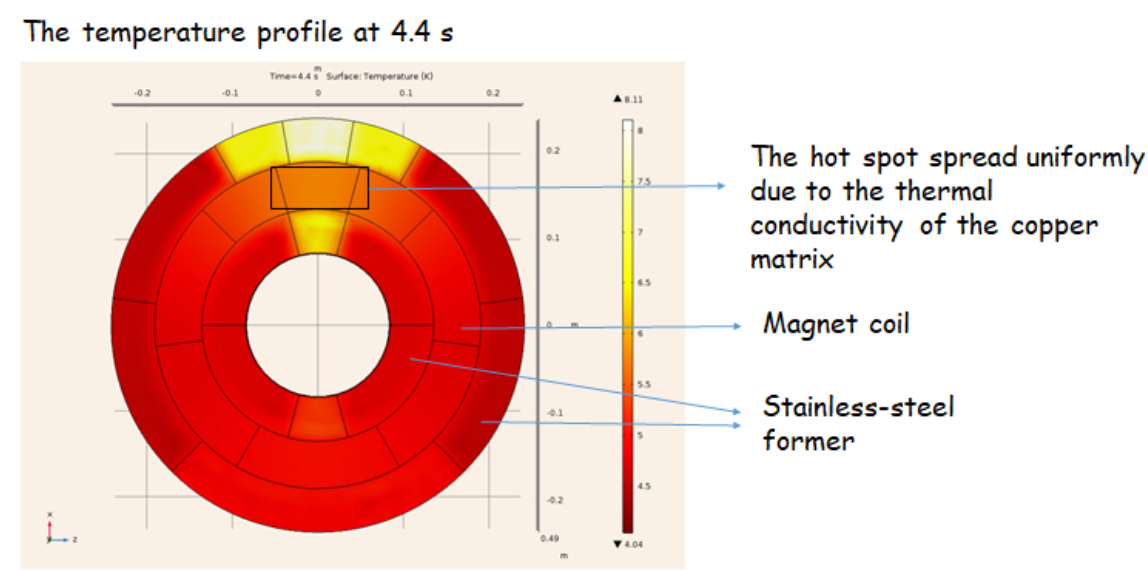

Figure 8: The temperature distribution in the superconducting magnet and the stainless steel former at the end of the beam spill $(t=4.4 \mathrm{~s})$ with the beam intensity of $1 \times 10^{12}$ proton/sec and no pumping on the magnet reservoir.

The liquid helium temperature can be lowered by pumping the helium vapor out of the magnet 
reservoir. Figure 9 shows the relation between the vapor pressure and the liquid helium temperature for ${ }^{3} \mathrm{He}$ and ${ }^{4} \mathrm{He}$. The KNF-N0150 pump has the capability to lower the helium temperature down to $2.5 \mathrm{~K}$ and hence, provide a chance to increase the beam intensity.

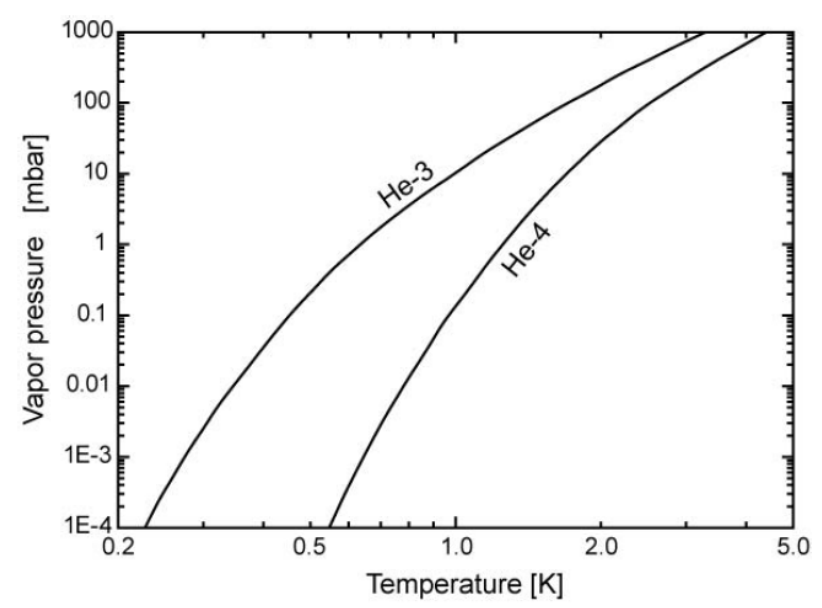

Figure 9: The vapor pressure versus temperature of the ${ }^{4} \mathrm{He}$ and ${ }^{3} \mathrm{He}$. Image source [22]

Figure 10 shows the maximum temperature in the magnet as a function of time with the beam intensity is $3.2 \times 10^{12}$ proton/sec and the KNF-N0150 pump is running. The maximumtemperature peak is $6.1 \mathrm{~K}$ which is close to the quench threshold. Therefore, we can conclude that pumping out the helium vapor give us a room to increase the beam intensity up to $3.2 \times 10^{12}$ proton/sec.

\section{Conclusions}

We have successfully developed a numerical method to predict the temperature profile of the superconducting magnet, used for the SpinQuest experiment at Fermilab. In the method, the thermal conduction among the elements of the magnet, the external heat sources and the heat flow to the liquid helium were taken into full consideration. The governing differential equations were solved using Finite Element Method, implemented in COMSOL Multiphysics. We have also built a threedimensional map of the magnetic field inside and around the superconducting magnet, applying the same FEM method, which is crucial for quench-threshold determination. The main objective of this study is to calculate the maximum beam intensity before the magnet quench. We found that by pumping the helium vapor out of the magnet reservoir using KNF-N0150 pump we could afford $3.2 \times 10^{12}$ proton/sec of the proton beam without quenching the superconducting magnet. 


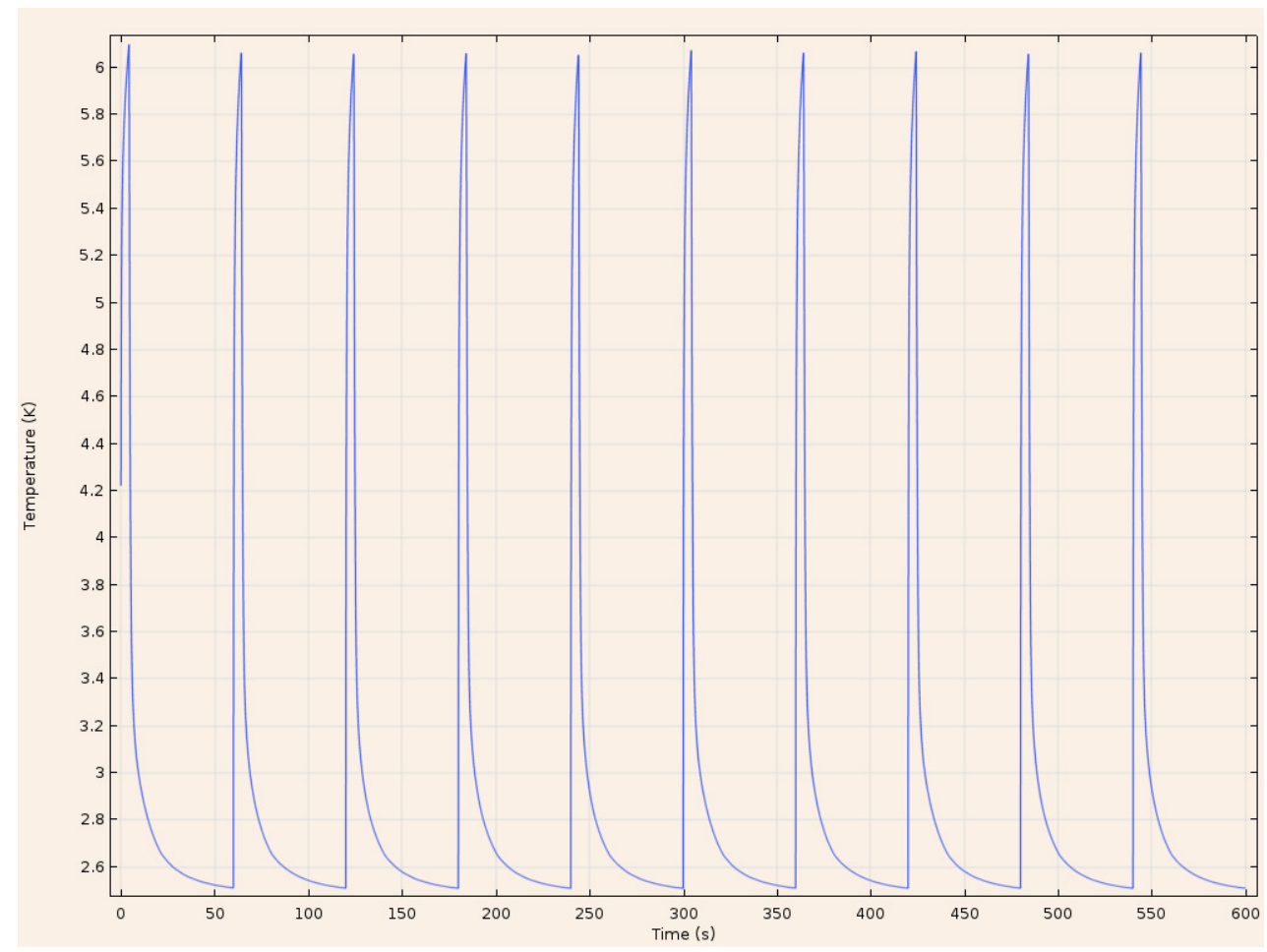

Figure 10: The maximum temperature in the magnet with the increase of beam intensity up to $3.2 \times 10^{12}$ proton/sec. 


\section{References}

[1] R. L. Jaffe and A. Manohar, Nucl. Phys. B 337, 509 (1990).

[2] C. A. Aidala, S. D. Bass, D. Hasch and G. K. Mallot, Rev. Mod. Phys. 85, 655 (2013).

[3] A. Deshpande, Prog. Theor. Exp. Phys. 2015, 03A107.

[4] K. F. Liu et al., PoS LATTICE 2011, 164 (2011).

[5] D. Sivers, Phys. Rev. D41, (1990) 83.

[6] A. Airapetian et al. [HERMES Collaboration], Phys. Rev. Lett. 103, 152002 (2009).

[7] M. Alekseev et al. [COMPASS Collaboration], Phys. Lett. B673, 127 (2009).

[8] X. Qian et al. [JLab Hall A Collaboration], Phys. Rev. Lett. 107, 072003 (2011).

[9] A. Klein, D. Keller, spokesperson, "SeaQuest with a Transversely Polarized Target (E1039) ," (https://pdfs.semanticscholar.org/898a/cc92285cf39584018107a8af531e25d06c38.pdf), Proposal to Department of Energy.

[10] C. Keith, Private communication.

[11] P. Bauer. "Stability of Superconducting Strands for Accelerator Magnet," Doctoral Dissertation (1998), TU Wien.

[12] G. Willering. "Stability of Superconducting Rutherford Cables,” Doctoral Dissertation (2009), University of Twente.

[13] D. Paudel. "Quench Simulation of Superconducting Magnets with Commercial Multi-Physics Software," Master Thesis (2015), Aalto University.

[14] J. Y. Jang, Y. J. Hwang, M. C. Ahn and Y. S. Choi, Physica C: Superconductivity and its application 550, (2019) 99-106.

[15] K. Pietrak, and T. S. Wisniewski, Journal of Power Technologies 95 (1), (2015) 14-24.

[16] E. Haro. ”Simulation Tool Development for Quench Modelling,” Doctoral Dissertation (2016), Tampere University of Technology.

[17] L. E. Evseeva and S. A. Tanaeva, Cryogenics 35, (1995) 277-279.

[18] S. Kelham and H. M. Rosenberg, J. Phys. C: Solid State Phys 14, 1737 (1981).

[19] S. Russenschuck (2011). "Field Computation for Accelerator Magnets," John Wiley \& Sons, Ltd.

[20] L. Rossi and M. Sorbi, "MATPRO: A Computer Library of Material Property at Cryogenic Temperature," CARE-Note-2005-018-HHH (2006).

[21] P. Bauer, H. Rajainmaki and E. Salpietro, "EFDA Material Data Compilation for Superconductor Simulation," EFDA Memo (2007).

[22] K. Hock, “Cooling Techniques,” Lecture notes of PHYS393, University of Liverpool (2011). 